Sätze der von diesem aufgestellten Gebühren für Ingenieure 1920 , soweit sie für Chemiker in Betracht kommen, insbesondere Absatz IV, Gebühren für Sachverständige usw. Leistungen, ferner für Leistungen nach der Zeit, ebenfalls als „üblicher Preis" auch für Chemiker anzusehen sind.

Hierzu ist ebenfalls als Beleg Nr. 13 der Zeitschrift für angewandte Chemie beigelegt, und zwar wird auf Seite 56 verwiesen. wollen.

Ich bitte mir diese Anlagen später wieder zurïckgeben zu

Darauf erging nun folgender Beschluß des Landgerichts Kassel: BeschluB.

In sachen der Firma D R. ........

hat die 3. Zivilkammer des Landgerichts Kassel auf die Beschwerde des als Sachverstïndigen gehörten Chemikers Dr. K. Brauer in Kassel gegen den Beschlußs des Amtsgerichts, Abtl. 16 in Kassel vom 21. November 1920 in der Sitzung vom 5. März 1921 beschlossen:

Unter Aufhebung des angefochtenen Beschlusses wird die Entschädigung des Sachverständigen Dr. K. Brauer in Kassel auf M 152,60 festgesetzt.

Grüinde.

Der Beschwerdeführer hatte für ein in der oben bezeichneten Sacbe erstattetes Gutachten Gebühren in Höhe von M 152,60 liquidiert. Das Amtsgericht Kitssel, Abtl. 16, setzte die Entschädigung des Sachverständigen auf M 48,40 gemäß $\$ \$ 3,13$ Geb.-Ordnung für Zeugen und Sachrerständige fest, in der Erwägung. daß die Leistung des Sachverständigen nicht als besonders schwierig anzusehen sei und daher nur eine Höchstgebühr von M 7,50 für die Stunde dem - Sachverständigen zustehe und daß ferner die Hinzuziehung eines Assistenten bei der Einfachheit der Sache nicht erforderlich gewesen sei. Gegen diesen Beschluß hat der Sachverständige Beschwerde eingelegt und ersucht, die Entschädigung seines ursprünglichen Antrags gemäb festzusetzen. Fr beansprucht die Vergütung des üblichen Preises nach $\$+$ der Geb-ord. für Zeugen und Sachverständige. $Z$ war besteht fïr (hemiker eine besondere Taxvorschrift in dem Tarif für Chemiker (Ges. vom 14. Juli 1909 G. S. 625), jedoch kann der Sachverständige an Stelle der Taxvorschriften die Berechnung der Gesamtvergütung nach den Vorschriften der Geb.Ord. für Zeugen und Sachverständige fordern (\$13 Abs. 2 Geb.-Ord. für Zeugen und Sachverständige). Der Beschwerdeführer kann daher mit Recht die Gewährung des üblichen Preises nach \& 4 Geb.-Ord. für Zeugen und Sachverstiindige verlangen.

Die in dem vom Beschwerdeführer vorgelegten Gebührenverzeichnis enthaltenen Sälze, die von der Vereinigung württembergischer Nahrungsmittelchemiker in Mïr 1920 aufgestellt sind, sind von dem Verein deutscher Chemiker als übliche anerkannt worden und zwar mit einem Aufschlag von $50^{\circ}$ (vgl. Zeitschrift für angewandte Chemie Ni. 55 vom 17. September 1920 und Nr. 13 vom 15). Febr. 1921. Diese Sätze sind sonach als „übliche" Preise im Sinne des Gesetzes anzusuhen. Als Stundenverguitung kann darnach für die erste Stunde M 40 , für jede weitere Stunde M 15 berechnet werden.

Der Sachverständige hat Vergütung für 7 Stunden verlangt und kann dafür M 40 (fürr die erste und M 90 [förmlich je M 15 für die weiteren sechs Stunden] - M 130 und 50\% Aufschlag) M 65 also M 195 berechnen. Beansprucht hat er nur eine Gebühr von M 152,60. Diese war ihm demnach zizubilligen.

Ausgefertigt: Kassel, den 23. Mä\% 1921.

Unterschrift.

Hierdurch sind also tatsïtchlich dif vom Verein deutscher Chemiker und dem Verbande selbständiger offentlicher Chemiker zugrundegelegten Gebühren mit $500_{10}$ Aufschlag als "übliche“ ausdrücklich anerkannt. Ich halte diese Entscheidung für so wichtig, daß ich sie hier veröffentliche, so daß Kollegen in ähnlichen Fällen sich darauf beziehen können.

Bei dieser Gelegenheit sei noch auf folgenden merkwürdigen Umstand bei der Besoldung chemischer Sathverständiger hingewiesen.

Bei Wahrnehmung von Terminen kann bekanntlich nicht der oben mehrfach erwähnte ,übliche" Preis in Anrechnung gebracht werden, sondern es heißt im $\$ 4$ der Gebührenordnung für Zeugen und Sachverstiindige, letzter Absatz, folgendermalien

„Beschränkt sich die Tätigkeit des Sachverständigen auf die Teilnahme an Terminen, so erhält er lediglich die im $\$ 3$ bestimmte Vergütung*.

Mlan kann also demnach einen Satz von M 7,50, höchstens M 15,die Stunde liquidieren, wobei der letzter Kostensatz, wie bereits oben ausgeführt, nur sehr selten bewilligt wird.

Nun gibt es noch eine besondere Taxvorschrift in dem Tarif für Chemiker, Medizinalheamte usw. (Gesetz vom 14. Juli 1909). Danach hatte der Chemiker bisher bei Wahrnehmung eines Termins bis zu zwei Stunden $\mathrm{M}$ 6,- zu beanspruchen, wozu laut Verordnung vom 11. März $1920^{1}$ ) $100^{\circ}$. Zuschlag kamen, so daß der Betrag M 12,ausmachte. Wenn nun ein Termin zwei Stunden dauert, bekam der

1) Erlaß des Ministeriums für Volkswohlfahrt, betreffend Änderung des Tarifs für die Gebiihren der Kreisärzte sowie des Tarifs für die Gebübren der Chemiker für gerichtliche und medizinalpolizeiliche Verrichtungen (I MI 561). Zeitschr. f. Unters. d. Nahrungs- u. Genußmittel Bd. 41 1921, Heft $3 / 4$ Abtl. Gesetze, Seite : chemische Sachverständige nach dieser Vorschrift nur M 12,-, während er nach $\& 3$ der Gebulhrenordnung für Zeugen und Sachverständige zwei Stunden zu mindesten M 7,50 - also M 15, _ - vergütet bekam. Er kam also bei Entlohnung nach der letzteren Gebührenordnung besser fort. In nevester Zeit ist nun die Sache gerade wieder umgekehrt. Die Taxvorschriften für gerichtliche und medizinalpolizeiliche Untersuchungen vom 14. Juli 1909 sind inzwischen in Preußen um $300 \%$ erhøht worden ${ }^{2}$ ), so daß der Chemiker für einen Termin bis zu zwei Stunden M 24, - erhält, während er nach \&3 der Gebührenordnung für Zeugen und Sachverständige bei Dauer eines Termins von einer Stunde nur M 7,50 oder höchstens M 15,-- erhält. Jetzt kommt er also wieder bei der anderen. Taxvorschrift besser fort. So erging es mir kürzlich bei einem Termin. Ich beanspruchte laut $\$ 3$ der Gebührenordnung für Zeugen und Sachverständige M 15, - für eine Stunde Zeitversäumnis. Sie wurde mir nicht bewilligt, weil es sich nach Ansicht des Gerichts nicht um eine besonders schwierige Leistung handelte. Dagegen wurden mir M $24,-$ zugesandt auf Grund der um $300 \%$ erhöhten anderen Taxvorschrift, da man danach für jeden Termin mindestens M $24,-$ bekommt!

Man sieht also, zu welch unerquicklichen Zuständen das Bestehen zweier verschiedener Gebührenordnungen für Chemiker wenigstens in Preußen führen kann.

Wenn eine Gebührenordnung erhöht wird, so muß doch unbedingt auch die andere erhöht werden, um eine gleichmäßige Berechnung durchzuführen.

Die Zustände, daß3 der Chemiker sich nach Wunsch einmal nach der einen oder nach der anderen Gebührenordnung entlohnen lassen kann, sind doch unhaltbar, denn es dient zur Benachteiligung desjenigen, der gerade nicht im Bilde ist, welche von beiden Gebührenordnungen nun augenblicklich giinstiger ist als die andere und das wechselt ja, wie vorstehendes Beispiel zeigt.

[A. 60.]

\section{Zur Reform der Ausbildung der Chemiker.}

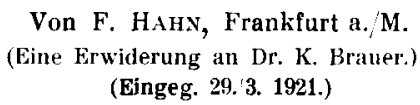

Die Ausführungen des Herrn $W u l f f$ in Nr. 11 dieser Zeitschrift schienen als Anregung beachtenswert, nach der Fortsetzung aber, die sie in Nr. 23 durch Herrn Dr. Brauer gefunden haben, halte ich eine Entgegnung für notwendig, selbst auf die Gefahr hin, daß Herr Dr. Brauer, dessen Verdienste auf praktischem und wissenschaftlichem Gebiet ich kenne und schätze, auch mir die Vertretung "einseitiger Professorenweisheit und privater Interessen der Großindustrie" vorwirft.

Strittig zwischen uns ist im wesentlichen die Frage: Ist die von manchen (nicht „den “) Studierenden gewünschte überwiegend praktische Ausbildung wirklich die erforderliche? Soll der Student in allgemeinen schon auf der Universität „noch etwas wirklich Praktisches lernen"? Schadet es etwas, wenn er nicht weil3, "wie man den einfachsten Handelsartikel untersucht, wie man feststellt, ob ein Öl für eine Maschine brauchbar ist oder nicht", ,wie man wirklich ein Produkt, z. B. eine Schuhkreme untersucht, um seine Bestandteile herauszubekommen"? Diese Frage verneine ich aus einer oroßen Unterrichtserfahrung heraus. Nach meiner Auffassung genügt es vollkommen, wenn ein Student nach beendeter Universitätsausbildung imstande ist, eine technische Untersuchungsvorschrift mit Verständ$n$ is durchzulesen. Als Stubengelehrter wird er dann noch nicht fähig sein, sie nachzuarbeiten. Das mag ein Mangel sein, aber ich würde es für einen noch weit größeren Mangel halten, wenn er als frisch ausgebildeter Mann der Praxis zwar die Analyse eines Düngemittels einwandfrei durchführen könnte, dafür aber nicht wüßte, wie man aus dem Volumen des entwickelten NO unmittelbar den Gehalt an $\mathrm{NaNO}_{3}$ oder $\mathrm{NO}_{3}$ ' oder $\mathrm{N}_{2} \mathrm{O}_{3}$ berechnet. Wenn er zwar genau wüßte, wie man citratlösliche Phosphorsäure bestimmt, aber nicht warum. Manche Analysenmethode, nach der wir die Studenten noch arbeiten lassen, mag in der Praxis längst durch eine schneller und billiger auszuführende ersetzt sein. Aber diese technische Methode ist oft nur in einem Sonderfall anwendbar und liefert nur unter Benutzung eigens hierzu verwendbarer Apparate oder Innehaltung ganz bestimmter, durch Probieren ermittelter Arbeitsbedingungen brauchbare Resultate. Der angehende Analytiker soll jedoch vor allem solche Verfahren kennen lernen, die einermöglichst allgemeinen Anwendung fähig sind, und bei denen sich die einzuhaltenden Bedingungen durch theoretische Überlegungen auffinden oder zum mindesten verstehen lassen. Ist er so ausgebildet, dann wird es ihm nicht schwer fallen, auch die technischen Verfahren - natürlich nicht aus Büchern, sondern unter sachkundiger Leitung - rasch und mit Verständnis sich anzueignen. Gelingt ihm dies nicht, so ist entweder seine allgemeine Durchbildung oder die pädagogische Befähigung des leitenden Praktikers mangelhaft. Das weiß die Großindustrie; sie hat längst die Erfahrung gemacht, daß sich nicht die Anfünger am besten einarbeiten und dauernd bewähren, die schon etwas Praktisches können, sondern die, bei denen die allgemeine wissenschaftliche Grundlage am solidesten ist. Deshalb und nicht aus "privater Interessenpolitik“ wünscht sie, daß die Studenten nicht schon während des Studiums in einzelnen Zweigen

2) Erlaß wie oben vom 20. Dez. 1920, ebenda Seite 7 
der chemischen Praxis ausgebildet werden. Denn nur wenige Studierende sind fähig, neben der wissenschaftlichen Ausbildung die prak tische zu pflegen, die größte Gefahr aber, der wir zutreiben könnten, wäre die, daß wir statt der wissenschaftlichen die praktische betreiben, da 3 wir Laboranten statt Chemiker heranbilden.

Für Studenten aber, die sich beides zugleich zumuten können, gibt es einen äußerst günstigen Weg, schon während des Studiums mit der Praxis in Berührung zu kommen: Ferienarbeit im praktischen Betrieb selbst. Es ist dringend zu wünschen, daß alle Leiter geeig neter chemischer Betriebe hierzu im reichsten MaRe Gelegenheit schaffen. Es erscheint durchaus denkbar, daß solche Praktiker, die dies reichlich und mit Erfolg tun, in irgendeiner Form dem Lehrkörper der Hochschulen angegliedert werden; sofort ließe sich ein Zusammengehen zwischen Praxis und Wissenschaft dadurch erreichen, daß die Vermittlung der Ferienarbeit den Hochschulinstituten übertragen würde. Es wäre ein Anreiz für die Studenten, sich die Möglichkeit zur praktischen Arbeit in einem Teil der Ferien durch verständige wissenschaftliche Arbeit im Semester zu sichern, und es läge für die Betriebe hierin eine Gewähr, nur solche Studenten zugewiesen zu bekommen, die nach kurzer Einarbeitung auch wirklich verständige und wertvolle Arbeit leisten können. Die Studenten werden hierbei die Wechselwirkung zwischen Wissenschaft und Praxis und die Praxis selbst viel besser kennen lernen als durch das noch so gute Kolleg eines Praktikers. Die Praxis mit allen ihren großen Wundern: straffes und zielbewuBtes Arbeiten, beste Ausnutzung von Raum, Zeit, Material usw. Sie werden dabei aber auch die Nachteile der Praxis kennen lernen: die strenge Arbeitsteilung und die gewaltige Belastung, die viele Praktiker daran verhindert, auch nur etwas über den Tagesbedarf hinaus zu schaffen und zu streben. Selbst wo das Streben aber vorhanden ist, da wird im Drange der Berufsarbeit vielfach die Übersicht und der Blick für innere Zusammenhänge verlorengehen, wenn nicht der wissenschaftliche Grund besonders fest war. ${ }^{1}$

Wenn das ein Student bei einem Ferienausflug in die Technik bemerkt und ihn diese Wahrnehmung veranlafst, die kurze Zeit der akademischen Lernfreiheit recht auszunützen, um sich ein möglichst reiches und tiefes theoretisches Wissen zu erwerben, dann wird die Praxis zwar nicht praktisch geschulte, aber praktisch brauchbare Anfänger bekommen. Gerade wenn wir die Industrie für die harte Zeit des Wiederaufbaus stärken wollen, müssen wir zwar auch dafür sorgen, daß mehr als bisher alte Praktiker in die Wissenschaft, vor allem aber dafür, daß ebenso wie bisher junge Wissenschaftler in die Praxis kommen.

\section{Neue Hochvakuummethoden in der Chemie.} Von M. Volmer.

luszıg aus einem Vortrag im Hamburger Bezirksverein des Vereins deutscher Chemiker (Eingeg. 31.;3. 1921.)

\section{A. Pumpen.}

Die Technik der Hochvakuumerzeugung hat in den letaten Jahren außerordentliche Fortschritte gemacht. Wenn auch das Hauptinteresse für diese Entwicklung auf physikalisch-technischem Gebiet liegt, so lassen sich die modernen Mittel auch für chemische Zwecke dienstbar machen und ermöglichen neue Arbeitsmethoden. Es sollen hier nach einer kurzen Besprechung der Pumpen einige Anwendungen auf chemische Fragen ausgeführt werden.

Die große Mehrzahl der älteren Pumpen ist mit Ausnahme der Strahlpumpen im Prinzip der alten Guericke-Pumpe gleichartig, sie beruhen auf der Verdrängung der Luft durch mechanische Mittel. Während alle früheren Erfindungen auf dem Pumpengebiet im wesentlichen auf die Vervollkommnung dieser Methoden hinarbeiten, indem sie die für die Hochvakuumerzeugung hinderlichen Fehler, insbesondere den schädlichen Raum möglichst klein zu machen suchen, ist es das Verdienst von W. Gaede, nachdem er selbst zunächst diesen Weg erfolgreich beschritten hatte (Hg-Gaedepumpe, Kapselpumpe, Stiefelpumpe), ganz neue Prinzipien für die Hochvakuumerzeugung nutzbar gemacht zu haben. So entstand als erste die Molekularluftpumpe, bei der er die äußere Reibung der Gase benutzt, um sie mit Hilfe einer schnell rotierenden Walze fortzuschaffen. Dieser Pumpe, die seinerzeit sowohl durch ihre überraschende Sauggeschwindigkeit und Höhe des erreichbaren Vakuums als auch durch ihre erstaunliche technische Durchbildung allgemeines Aufsehen erregte, folgte dann eine frappierend einfache und doch in gewisser Hinsicht, nämlich was die Höhe des Vakuums anbetrifft, noch überlegene Vorrichtung, die Diffusionspumpe. Bei ihr wird eine Diffusionswand, d. h. eine Wand mit so kleinen Öffnungen, daß ihr Durchmesser der Größenordnung nach höchstens gleich der freien Weglänge der Moleküle ist, zur Absperrung des zu evakuierenden Raumes benutzt. Auf der entgegengesetzten Seite der Wand wird dauernd eine luftfreie Dampfatmosphäre aufrechterhalten. Durch Molekularbeengung treten dann die Luftmoleküle durch die Diffusionswand in den Dampfraum, bis der Partialdruck der Luft auf beiden Seiten gleich, d. h. Null geworden ist. Die entgegenwandernden

1) Man vergleiche z. B. den Aufsatz von Auerbach und Millbradt über die Verdunstung von Salmiakgeist in $\mathrm{Nr}$. 21 dieser Zeitschr. Dort werden teils ganz selbstverständliche Dinge experimentell bewiesen, teils längst widerlegte Ansichten (über Zusammensetzung und Natur des beim Verdunsten ron Salzsäure hinterbleibenden Rückstandes) als geltend vorgetragen.
Dampfmoleküle werden auf der anderen Seite durch Kühlen entfernt so daß der RAum theoretisch vollig entleert wird. Wesentlich für die Wirkung dieser Pumpe ist die Eigenschaft einer Diffusionswand, nur eine reine Molekularbewegung und keine Strömung der Gase zuzulassen, wodurch sie als Trägerin der Druckdifferenz zwischen Dampfraum und Hochvakuumraum dienen kann.

Leider war der technische Erfolg dieser geistreichen Erfindung nur von kurzer Dauer. Während in Deutschland der Krieg die Aufmerksamkeit auf andere Aufgaben lenkte, wurden, angeregt durch die $G$ a e d esche Idee, in Amerika von verschiedener Seite Versuche gemacht, die Leistung der Diffusionspumpe zu steigern. Dabei entstand eine neue Art von Quecksilberdampfpumpen, die in ihrer technischen Leistung die Diffusionspumpe weit hinter sich ließen. Diese in theoretischer und praktischer Beziehung von L a $\mathrm{g} \mathbf{m}$ u ir zuerst ausgearbeiteten Pumven stehen der letzteren verhältnismäßig fern und sind weitaus näher verwandt den älteren Wasserdampfstrahlpumpen. Wie bei diesen wird die mitführende Wirkung eines Dampfstrahls, speziell eines $\mathrm{Hg}$ Dampfstrahls zur Entfernung der Luft aus dem Rezipienten benutzt. Der Unterschied beider liegt einmal darin, daß bei den Wasserdampfstrahlpumpen, die bei hohen Drucken und entsprechend kleinen freien Weglängen der Moleküle arbeiten, die innere Reibung des abzusaugenden Mediums für den Effekt maßgebend ist, während bei den Hg-Hochvakuumdampfstrahlpumpen ähnlich wie bei der $\mathrm{G}$ a e d e schen Molekularluftpumpe die äußere Reibung wirksam wird. Von der Diffusionspumpe, mit der die neuen Pumpen vielfach auch heute noch durcheinandergeworfen werden, unterscheiden sie sich durch das Fehlen der Diffusionswand; die Druckdifferenz zwischen Vor- und Hochvakuum wird bei diesen durch die kinetische Energie des Strahles kompensiert Von den alten Dampfstrahlpumpen unterscheiden sich die Lang muir scben Pumpen noch in charakteristischer Weise dadurch, daß an der Zusammentrittsstelle von Dampfstrahl und Luft ein Kühler angeordnet ist, um ein Zurückströmen von $\mathrm{Hg}$-Dampf in das Hochvakuum (durch Reflexion der Hg-Moleküle an den ungekühlten Wänden), welches die Wirkung stören würde, zu verhindern (Kondensationspumpen). Ein näheres Eingehen auf die verschiedenen Pumpenkonstruktionen kann an dieser Stelle unterbleiben, da bereits zweimal eine zusammenfassende Darstellung des älteren Materials in vorzüglicher Weise durch Gehrts ${ }^{1}$ ) gebracht worden ist.

Die technische Leistung der Kondensationspumpen übertrifft die aller bis dahin bekannten Pumpen. Infolgedessen eroberten sie sich schnell ihren Platz in der einschlägigen Industrie, insbesondere wurden sie bereits unentbehrlich bei der Herstellung der Verstärker und Senderröhren der drahtlosen Telegraphie, bei denen ein Vakuum von etwa $10^{6} \mathrm{~mm}$ erforderlich ist. Auch für die Herstellung der Röntgenröhren haben sie sich bereits hervorragend bewährt.

Allerdings haben die Pumpen eine unangenehme Eigenschaft, sie erfordern nämlich $\mathrm{zu}$ ihrem Betrieb ein Vakuum von mindestens einigen Zehnteln Millimeter. Ein solches Vorvakuum ist aber mit einiger Sicherheit und Srhnelligkeit nur durch Anwendung von bereits zwei Pumpen, z. B. zwei Ölpumpen oder einer rotierenden Quecksilberpumpe in Verbindung mit der dazu nötigen Vorpumpe zu erhalten, wodurch die allgemeine Anwendung der neuen Pumpen recht erschwert wird. Bereits vor vier Jahren bemühte ich mich gelegentlich einer Arbeit, bei der ich die Gaedesche Diffusionspumpe benutzte, dieses unbequeme Druckgebiet von $20-0,1 \mathrm{~mm}$ mit Hilfe von Quecksilberdampfstrahlpumpen zu überbrücken. Diese Versuche führten zur Konstruktion der Hg-Dampfstrahlpumpe, die vor einiger Zeit beschrieben wurde. Die Pumpe übertraf die anfangs beabsichtigte Wirkung insofern, als sie allein bereits ein Vakuum von ca. $0,001 \mathrm{~mm}$ zu erreichen gestattete, und ein für chemische $Z$ wecke brauchbares Hilfsmittel für Arbeiten bei niederen Drucken lieferte. Es sind seitdem von verschiedener Seite Versuche unternommen worden, die Wirksamkeit der Pumpe weiter zu steigern. Doch blieben diese Bestrebungen entweder ohne nennenswerten Erfolg oder sie führten zu sehr komplizierten Apparaten, deren Kosten sich höher stellten als die einer Kombination der älteren Pumpe mit einer Langmuirschen Kondensationspumpe, die wiederum in der Leistung erheblich uberlegen war. Eine dankbare Aufgabe, die gemeinsam mit der Firma Hanff \& Buest in Angriff genommen wurde, schien uns darin zu bestehen, die Leistung der $\mathrm{Hg}$ Dampfstrahlpumpen für höhere Drucke und gleichzeitig ihre Betriebssicherheit so zu steigern, daß sie für technische Zwecke herangezogen werden können. Die eingehende Berücksichtigung der Strömungsgesetze sowie die Verwertung rein praktischer Erfahrung führten dazu, Pumpen von äußerst einfacher Bauart zu schaffen, deren Leistung das Vielfache der bisherigen war und deren Vakuumhöhe unbegrenzt war.

Bei diesen Arbeiten war gleichzeitig erkannt worden, daß es stets von großem Vorteil ist, die Pumpleistung auf mebrere Stufen zu verteilen, weil bei unwesentlich gesteigerter Heizung eine ganz erhebliche Erhőhung der Betriebssicherheit gegenüber Schwankungen des Vorvakuums erreicht wurde. Insbesondere zeigte sich der Vorteil der mehrstufigen Pumpen bei Verunreinigungen. Alle Hg-Dampfstrahlpumpen sind empfindlich gegen gewisse Verunreinigungen, z. B. Fettdämpfe, welche in die Pumpe hineingelangt, ihre Wirkung weitgehend herabsetzen. Während bei einstufigen Pumpen die Verunreinigungen sich gerade an der wirksamen Stelle, nämlich an der der Zusammentrittsstelle von Dampfstrahl und Luft zunächstliegenden Kühlerwand ansammeln, werden sie bei zweistufigen Pumpen bis zur Vorstufe geschafft

1) Naturwissenschaften 7, 983 [1919]. Z. f. techn. Phys. 1, 68 [1920]. 